\title{
The ISOLDE Facility: recent highlights and the HIE- ISOLDE project
}

\author{
Maria J. G. Borge ${ }^{1}$ \\ ISOLDE, CERN, \\ Ch-1211-Geneva-23, Switzerland \\ mgb@cern.ch
}

The ISOLDE facility at CERN has as objective the production, study and research of nuclei far from stability. The facility provides low energy radioactive beams and post-accelerated beams. In the last 45 years the ISOLDE facility has gathered unique expertise in research with radioactive beams. Over 700 isotopes of more than 70 elements have been used in a wide range of research domains, including cutting edge studies in nuclear structure, atomic physics, nuclear astrophysics, and fundamental interactions. These nuclear probes are also used to do frontier research in solid state and life sciences. There is an on-going upgrade of the facility, the HIE-ISOLDE project, which aims to improve the ISOLDE capabilities in a wide front, from an energy increase of the post-accelerated beam to improvements in beam quality and beam purity. The first phase of HIEISOLDE will start for physics in the autumn of 2015 with an upgrade of energy for all postaccelerated ISOLDE beams up to $5.5 \mathrm{MeV} / \mathrm{u}$, the second phase will increase the energy until 10 $\mathrm{MeV} / \mathrm{u}$. Plans exist of complementing the facility with a highly performing Storage Ring, TSR presently at Heidelberg in Germany. The addition of the TSR will allow the beams from HIEISOLDE to be stored and cooled, providing much greater luminosity and much better beam definition. In this contribution the most recent highlights of the facility and an overview of the approved day-one experiments for HIE-ISOLDE phase I are presented.

X Latin American Symposium on Nuclear Physics and Applications (X LASNPA)

December 1-6 2013

1

Speaker on leave from Instituto de Estructura de la Materia, CSIC, Serrano 113bis, Madrid, Spain 


\section{The ISOLDE Facility}

The Isotope Separator On-Line, ISOLDE, is a facility dedicated to the production of a large variety of radioactive ion beams for many different experiments in the fields of nuclear and atomic physics, materials science and life sciences. The facility is located at the ProtonSynchrotron Booster (PSB) of the European Organization for Nuclear Research, CERN. The beam delivered by the PSB injector is pulsed and contains up to $3.10^{13}$ protons/pulse with a spacing of $1.2 \mathrm{~s}$ or more, giving a typical average proton current on target of $2 \mathrm{~mA}$ [1]. The reaction products are stopped in the bulk of the target material, thereafter transported to an ion source and accelerated again.

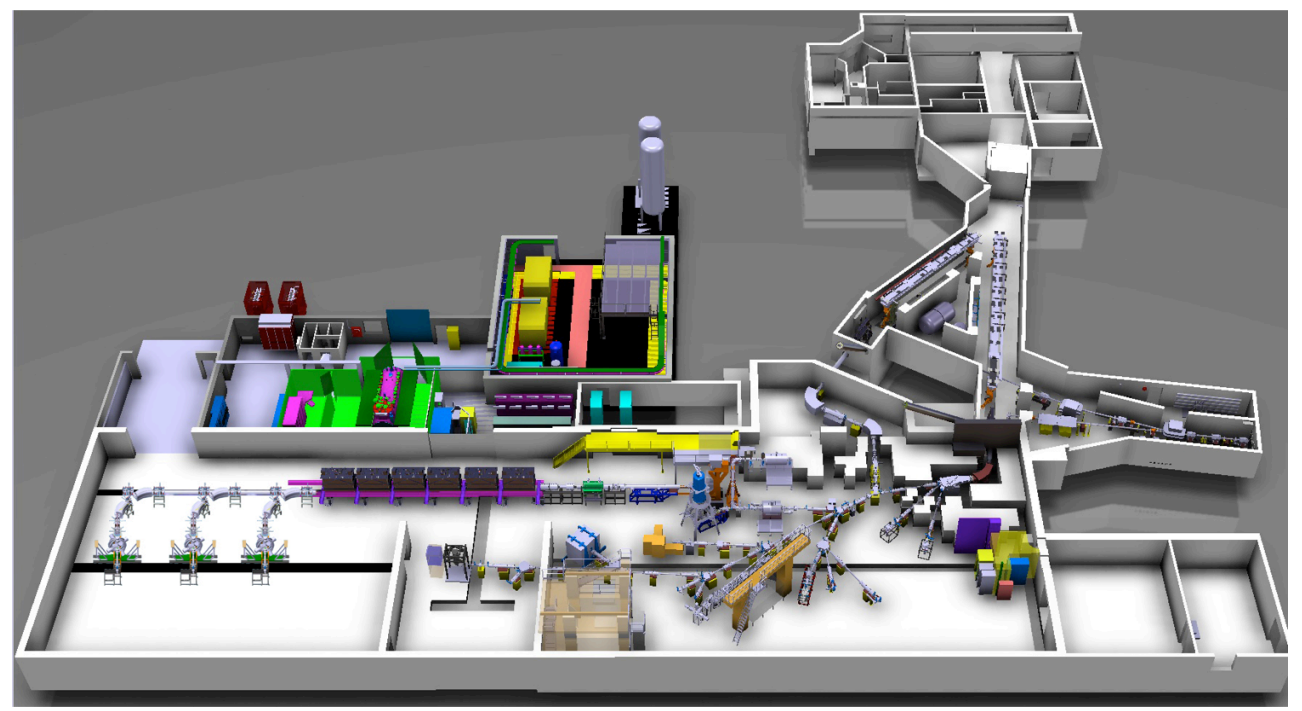

Fig. 1 Layout of the ISOLDE facility. The new HIE-ISOLDE linac and the low energy beam lines are displayed.

More than 20 different target materials and ionizers are in use. The target material is kept at a temperature between 1000 and 2000 degrees so that the radioactive atoms produced diffuse out of the target into different dedicated ion sources. Ionization can take place in hot plasma, on a hot surface or by laser excitation. Chemical selectivity is obtained by the right combination of target-ion sources giving rise to a selective production of isotopes of more than 70 of the chemical elements. The knowledge accumulated over more than forty five years on how to construct targets and ion sources tailored to release pure beams of specific elements is one of ISOLDE's strong points. After extraction and acceleration to $30-60 \mathrm{kV}$ the ions are sent to the magnetic mass separators. ISOLDE uses two different mass separators, one the so-called General Purpose Separator (GPS) with a mass resolving power, M/DM, of more than 1000, and the other, a High Resolution Separator (HRS), which mass resolution is larger than 5000. The GPS and the HRS separators are connected to a common beam-line system. This allows for the optimization of the space and the flexibility of operation as the ions can be produced from two different target-ion source units. Figure 1 shows a drawing of the ISOLDE facility including the new buildings serving the superconducting Linac. 
The post-accelerator, REX-ISOLDE, in operation since 2001, has opened new fields of research in particular in reaction studies of light-medium mass nuclei with energies up to 3 $\mathrm{MeV} / \mathrm{u}$. A thorough technical description of the REX accelerator can be found in [2]. The singly charged ions from ISOLDE are captured and bunched in a large acceptance Penning trap (REXTRAP) and charge bred in the REXEBIS ion source to a large $\mathrm{A} / \mathrm{q}$ ratio, presently between 2 and 4.5. The higher charge of the beam allows it to be efficiently accelerated in a compact linear accelerator. REX is being upgraded (HIE-ISOLDE) to provide in average a maximum energy of $5.5 \mathrm{MeV} / \mathrm{u}$ in 2015. Radioactive beams of these energies will allow reaching the Coulomb barrier threshold for the wide range of nuclei available at ISOLDE. In a second phase of HIE-ISOLDE energies up to $10 \mathrm{MeV} / \mathrm{u}$ for the radioactive beams will be obtained.

In the following some of the recent highlights of ISOLDE will be presented.

\section{ISOLDE Highlights}

The CERN Council approved the ISOLDE facility 50 years ago in December 1964. With more than 45 years of activity, 20 of them at the PS-Booster, ISOLDE is still growing and attracts presently more than 450 researchers working on 90 experiments, with a rate of 50 experiments taking data per year. This radioactive beam facility has pioneered many achievements both at the level of designing new devices and of producing frontier Physics. Over the years the experiments of the ISOLDE facility have produced many valuable results, but 2013 was really exceptional. Three papers published within one month in the prestigious Nature journals have crowned the remarkable results obtained along the years. Due to the limited space I will concentrate my contribution in describing this high impact results.

\subsection{Determination of the Ionization Potential of Astatine}

The RILIS (resonant Ionization Laser Ion Source) lasers photo-ionise the neutral products of the proton induced nuclear reactions if the lasers are tuned to the right frequency. The atoms are ionized in two or three steps either via an auto-ionising state or directly to the continuum. The RILIS team in their quest to find new ionization schemes has been able to measure, for first time, the ionization potential of astatine, the least abundant element in nature, by laser ionization spectroscopy. The discovered series of high-lying Rydberg states enabled a high precision determination of binding energy of the valence electron of the astatine atom, the ionization potential (IP), to be 9.31751(8) eV [3]. The determined IP value serves as benchmark for quantum chemistry calculations of the properties of astatine. Further the determination of the IP of At is crucial for the predictions of atomic physics properties of the superheavy element $Z$ $=117$, its heaviest chemical homologue [3].

\subsection{Mass Measurements: Testing Magic Numbers in Calcium Isotopes}

The mass of a nucleus is together with its half-life and decay modes the first quantity to measure. The total binding energy of a nucleus contains great physics information and precision mass measurements often provide important tests of nuclear models. In the ISOLTRAP mass measurements the beam from ISOLDE is collected and bunched before being sent to the 
preparation trap where purification takes place. A detailed analysis of the ion motion in the precision trap shows that the cyclotron resonance of the ion can be determined through the measurement of the time of flight of the ion when ejected from the trap.

A pioneering work done at CERN thirty years ago [4] indicated that the magic numbers of. neutron and protons corresponding to closed shells could loose their special character due to the proton-neutron asymmetry. It is since then a great challenge for theory to predict the new magic numbers emerging far from stability, and closely related, to understand the different components of the strong force that act between neutron and protons. Most of the nuclear models predicted ${ }^{52} \mathrm{Ca}$ as a new double magic nucleus far from the valley of stability. The magic number character could be partially determined by measuring the mass and deducing the two neutron separation energy in the calcium isotopes around $\mathrm{N}=32$.

A multi-reflection time-of-flight mass separator (MR-TOF MS) was recently designed and installed at ISOLTRAP for isobaric purification. It has demonstrated a suppression of contaminants an order of magnitude better than conventional devices without relevant intensity and time losses. It has proven that it can be used for precision mass measurements on nuclides with ms half-lives and minute production rates and as an efficient and sensitive diagnostic station for laser spectroscopy. These developments have lead to the successful determination of the mass of neutron rich calcium isotopes at the verge of existence ${ }^{51-54} \mathrm{Ca}[5]$.

The determination of the ${ }^{51-52} \mathrm{Ca}$ masses was done using the ISOLTRAP Penning trap mass spectrometer. The previously described multi-reflection time-of-flight spectrometer was used for the first time for mass determination of the extremely low produced short-lived species ${ }^{53-}$ ${ }^{54} \mathrm{Ca}$. These measurements allow for the determination of the two-neutron separation energies, $S_{2 n}=B(Z, N)-B(Z, N-2)$, that is the difference of binding energy of two isotopes that differ in two neutrons. The $S_{2 n}$ is a sensitive probe of the evolution of nuclear structure with neutron number. The pronounced decrease in $S_{2 n}$ deduced from the data is well followed by the microscopic calculations using three nucleon forces based on chiral effective theory, and unambiguously establish $\mathrm{N}=32$ as a closed shell neutron number for neutron rich nuclei very far from stability. These results help to better understand dense nuclear matter and the subtle components of nuclear forces.

\subsection{REX-ISOLDE: Search for pear-shaped nuclei}

After a decade of physics with post-accelerated beams at ISOLDE [6] I will mention examples of the two major type of experiments done so far. In the energy range up to $3 \mathrm{MeV} / \mathrm{u}$ many reactions take place at or below the Coulomb barrier, so the main reaction mechanism is Coulomb excitation. The electromagnetic interaction is well understood and it is used as a probe of nuclear structure from the sub-barrier energies in Coulomb excitation experiments, "safe Coulex". This technique has experienced a renaissance in the last decade with the availability of post-accelerated beams. The key ingredient in these experiments is the gamma detection, and at ISOLDE the gamma spectroscopy of excited states and particle identification have been pushed forward with the MINIBALL/T-REX detector system. 


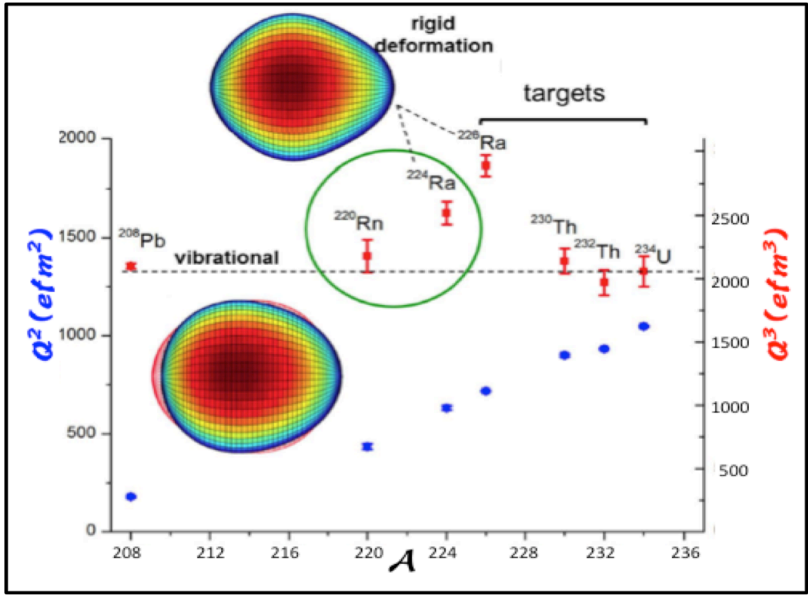

Fig. 2.- Systematics of the quadrupole and octupole moments for heavy nuclei with $\mathrm{A}=208-234$ including the new cases measured at ISOLDE ${ }^{220} \mathrm{Rn}$ and ${ }^{224} \mathrm{Ra}$. The present results indicate a clear evidence for strong octupole deformation in the latter [7].
The heaviest accelerated REXISOLDE beams of Radon and Radium nuclei were employed to investigate shape asymmetric configurations. Strong octupole correlations leading to pear shapes can arise when nucleons near the Fermi surface occupying states of opposite parity with orbital, D1, and total, DJ, angular momenta differing by $3 \hbar$. Pear shape nuclei have enhanced E1 and E3 transitions connecting rotational states of opposite parity. The E3 transition moments are collective in behaviour and insensitive to single-particle behaviour. Octupole correlations were studied by the determination of the electric octupole transition strengths in ${ }^{220} \mathrm{Rn}$ and ${ }^{224} \mathrm{Ra}$. The E3 moments are an observable that should provide direct evidence for enhanced octupole correlations for deformed nuclei. The measured E1, E2 or E3 transitions for ${ }^{220} \mathrm{Rn}$ and ${ }^{224} \mathrm{Ra}$ allowed the determination of the reduced matrix elements and the intrinsic moments. As shown on figure 2, the E2 moment, $\mathrm{Q}_{2}$, increases a factor of 6 from ${ }^{208} \mathrm{~Pb}$ to ${ }^{334} \mathrm{U}$ while the $\mathrm{E} 3$ moment, $\mathrm{Q}_{3}$, only varies up to $50 \%$ in the same region. The large values of $\mathrm{Q}_{3}$ measured for ${ }^{220} \mathrm{Rn}$ and ${ }^{224} \mathrm{Ra}$ point to an enhancement of octupole collectivity indicating the onset of octupole deformation in this region. While ${ }^{220} \mathrm{Ra}$ has $\mathrm{Q}_{3}$ values typical of an octupole vibrator, the $\mathrm{Q}_{3}$ values obtained for ${ }^{224} \mathrm{Ra}$ gives convincing evidence that this nucleus is of quadrupole-octupole shape in its ground state. Beyond the important input for models treating octupole deformation one can deduce from these data that the ${ }^{219,221} \mathrm{Rn}$ nuclei are not adequate candidates for atomic EDM measurements, as they are expected similar or weaker octupole collectivity than ${ }^{220} \mathrm{Rn}$. These results are summarized in a recent Nature paper [7].

Elastic, inelastic scattering, and transfer reactions yield important and unique information in the structure of exotic nuclei. The REX-ISOLDE beam energies restricted the studies to the light nuclei. Experiments were performed near the drip-lines where new phenomena were reveiled in the last decade. Elastic cross sections of halo nuclei near a strong electric field drastically changed at energies near the Coulomb barrier due to the loose character of the last nucleons. The elastic cross section of ${ }^{11} \mathrm{Be}$ and its core ${ }^{10} \mathrm{Be}$ was measured for the first time at ISOLDE [8]. The neutron transfer reaction of ${ }^{9} \mathrm{Li}+{ }^{2} \mathrm{H}$ was used to study ${ }^{8} \mathrm{Li}$ and to explore the unbound structure of ${ }^{10} \mathrm{Li}$, and the ${ }^{11} \mathrm{Be}(\mathrm{d}, \mathrm{p})$ reaction to characterize the excited states of ${ }^{12} \mathrm{Be}$ using Miniball for gamma detection [9]. 


\section{The HIE-ISOLDE Project}

The main features of the HIE ISOLDE project (HIE stands for "High Intensity and Energy) are to boost the energy of the beams, going in steps from currently $3 \mathrm{MeV} / \mathrm{u}$ via 5.5 $\mathrm{MeV} / \mathrm{u}$ to finally $10 \mathrm{MeV} / \mathrm{u}$. A factor of three in intensity is expected from the higher energy and intensity of the proton injector. In addition improvements in several aspects of the secondary beam properties such as purity, ionization efficiency and optical quality are addressed.

Since the call for proposals in October 2012 twenty-seven experiments with more than six hundred shifts have been approved for day-one physics. The physics cases approved expand over the wide range of post-accelerated beams available at ISOLDE. The increase in energy of the radioactive beams will enhance the cross section and the accessibility to detailed nuclear structure information at higher excitation energy. In the light nuclear region, reaction studies of astrophysical interest such as the search for high-excited states in ${ }^{8} \mathrm{Be}$ is planned to help solving the cosmological ${ }^{7} \mathrm{Li}$ problem. Nuclear structure studies are planned to characterize the cluster structure in ${ }^{10} \mathrm{Be}$ by transfer reaction. Further the unbound states in the proton-rich nucleus ${ }^{21} \mathrm{Al}$ will be explored by resonance elastic and inelastic scattering using the MAYA active target. For middle mass nuclei, the validity of a shell model description around ${ }^{78} \mathrm{Ni}$ will be studied and shape coexistence in the region $\mathrm{A}=70-80$ will be determined with high precision. Statistical properties of warm nuclei will be investigated by the low-energy enhancement of the gamma strength function of neutron rich nuclei. For heavier mass nuclei, quadrupole and octupole collectivity will be addressed in the neutron rich Te, Xe and Ba isotopes by Coulomb excitation, lifetime measurements and magnetic moment determination. Collective effects around the double magic ${ }^{132} \mathrm{Sn}$ will also be studied. For the heavier nuclei, shape coexistence in the light $\mathrm{Pb}$ isotopes will be explored, and measurements of octupole collectivity in the Rn and Ra nuclei using Coulomb excitation will continue. In the quest of super-heavies, it is proposed to investigate the influence of the predicted shell closures at $Z=120$ and $N=184$ by probing the height of the fission barrier. This will be achieved by exploring the contributions of quasifission and fusion-fission reactions; in particular the deformed ${ }^{95} \mathrm{Rb}$ beam on a ${ }^{209} \mathrm{Bi}$ target is expected to permit the study of these features. To learn more about the scientific opportunities see [10].

The proposed studies will be realised with the existing workhorses MINIBALL [11] and T-REX [12] plus new instrumentation for transfer reaction studies such as the active targets MAYA and the future ACTAR, a new general purpose scattering chamber and the two arms CORSET setup from GSI. Plans exist of complementing the facility with a highly performing Storage Ring, TSR presently at Heidelberg in Germany. The addition of the TSR will allow the beams from HIE-ISOLDE to be stored and cooled, providing much greater luminosity and much better beam definition. A HELIOS-type solenoidal spectrometer is envisioned to be located at the exit of the TSR. 
4.Summary

In summary, the future of ISOLDE is bright. It will restart in July 2014 with the low energy program. With more than 40 year of operation ISOLDE remains as the pioneer ISOLinstallation both at the level of designing new devices and production of frontier Physics.

Post accelerated beams up to $5.5 \mathrm{MeV} / \mathrm{u}$ for the wide range of nuclei produced at ISOLDE will be available from Autumn 2015. HIE-ISOLDE will be the only next-generation radioactive beam facility (as identified by the NuPECC LRP) available in Europe in 2015, and the most advanced ISOL facility world-wide.

References

[1] E. Kugler, Hyperfine Interactions 129 (2000) 23.

[2] The REX-ISOLDE Facility ed. by J. Cederkall et al, CERN Report, CERN-2005-009, (2005) 1.

[3] S. Rothe et al., Nature Communications. 43 (2013) 141.

[4] C. Thibaud et al., Phys. Rev. C 12 (1975) 644.

[5] F. Wienholtz et al., Nature 498 (2013) 346.

[6] P. V. Duppen and K. Riisager, J. Phys. G. Nucl. Part. 38 (2011) 02405.

[7] L. P. Gaffney et al., Nature 497 (2013) 199.

[8] A. Di Pietro et al., Phys. Rev. Lett 105, 022701 (2010).

[9] J. G. Johansen et al., Phys. Rev. C 88 (2013) 044619.

[10] HIE-ISOLDE: the scientific opportunities ed. by K. Riisager et al., CERN Report, CERN-2007-008. 